05,12

\title{
Динамика магнитного момента анизотропной наночастицы и плоской решетки в переменном поле
}

\author{
(C) А.М. Шутый, Д.И. Семенцов \\ Ульяновский государственный университет, \\ Ульяновск, Россия \\ E-mail: shuty@mail.ru \\ (Поступила в Редакцию 13 апреля 2018 г.)
}

Исследованы динамические режимы магнитного момента наночастицы с одноосной или кубической анизотропией. Выявлены различные по форме аттрактора и периоду регулярные колебания, режимы хаотической и квазипе-риодической динамики. Обнаружены состояния динамической бистабильности и получены переходы между входящими в бистабильность колебательными режимами с помощью импульсного изменения амплитуды переменного поля. Для сред с кубической анизотропией показано $90^{\circ}$-перемагничивание частицы при действии переменного поля. Выявлены параметры решеток наночастиц, при которых влиянием дипольдипольного взаимодействия на колебательные режимы магнитных моментов можно пренебречь.

Работа выполнена при поддержке Министерства образования и науки РФ в рамках государственного задания № 3.6825.2017/БЧ.

DOI: 10.21883/FTT.2018.12.46726.117

\section{1. Введение}

В последние годы ведется активное изучение магнитных наноструктур, представляющих магнитоупорядоченные решетки, состоящие из конечного числа однодоменных нанодиполей различной геометрии. Подобный интерес вызван прежде всего тем, что решетки размерности $1 D-3 D$ из ферромагнитных наночастиц рассматриваются в качестве наиболее перспективных сред для хранения и обработки информации [1-9]. Запись информации предполагает изменение ориентации магнитных моментов одной или нескольких нанодиполей локальным магнитным полем. Одним из методов считывания может рассматриваться резонансный, при котором соответствующий нанодиполь на частоте ферромагнитного резонанса возбуждается маломощным переменным полем, после чего производится регистрация отклика на этой частоте [10,11].

Кроме информационного аспекта, исследование решеток однодоменных наночастиц, связанных дипольдипольным взаимодействием, позволяет изучать в дипольном приближении коллективные эффекты в ансамблях с конечным числом частиц, которые представляются точечными диполями с магнитным моментом, пропорциональным их объему [12].

Дискретность структур приводит к существенным отличиям статических и динамических свойств подобных решеток от свойств макроскопических монодоменных объектов. К важным отличиям могут быть отнесены бистабильные состояния, обусловленные наличием различных ориентационных конфигураций с одинаковым суммарным магнитным моментом, а также возможность реализации управляемых переходов между данными конфигурациями и возникновение динамических колебательных режимов магнитного момента системы при ее перемагничивании [13-16].
В настоящей работе на основе численного анализа исследуются динамические режимы магнитного момента наночастиц с одноосной или кубической анизотропией, устанавливаемые под воздействием переменного магнитного поля. Для рассмотрения колебательных режимов в широком диапазоне параметров внешнего поля строятся бифуркационные диаграммы, позволяющие выявить не только области частот и амплитуд поля, отвечающие различным регулярным и хаотическим колебательным режимам, но и состояния динамической бистабильности. В работе рассмотрены также решетки наночастиц, с целью выявления условий, при которых колебания магнитных моментов составляющих ее элементов близки к колебаниям изолированных наночастиц.

\section{2. Исходные уравнения}

Рассмотрим наночастицу, находящуюся в однодоменном состоянии с магнитным моментом m. Будем считать, что наночастица имеет магнитную анизотропию либо одноосную (UA), либо кубическую (CA). При этом ось Y совпадает с легкой осью в случае UA, либо оси координат совпадают с легкими осями в случае СА. Уравнение Ландау-Лифшица, определяющее динамику моментного момента, записывается следующим образом [17]:

$$
\frac{\partial \mathbf{m}}{\partial t}=-\gamma \mathbf{m}\left(\mathbf{H}+\mathbf{H}_{a}\right)-\frac{\alpha}{m} \mathbf{m} \frac{\partial \mathbf{m}}{\partial t},
$$

где $\gamma$ - гиромагнитное отношение, $\alpha-$ параметр диссипации (далее принят равным $\alpha=0.01$ ), $\mathbf{H}$ - внешнее магнитное поле, а $\mathbf{H}_{a}$ - поле анизотропии. В случае UA поле анизотропии записывается в виде: $\mathbf{H}_{a}=k_{u} \mathbf{n}(\mathbf{m n})$, 

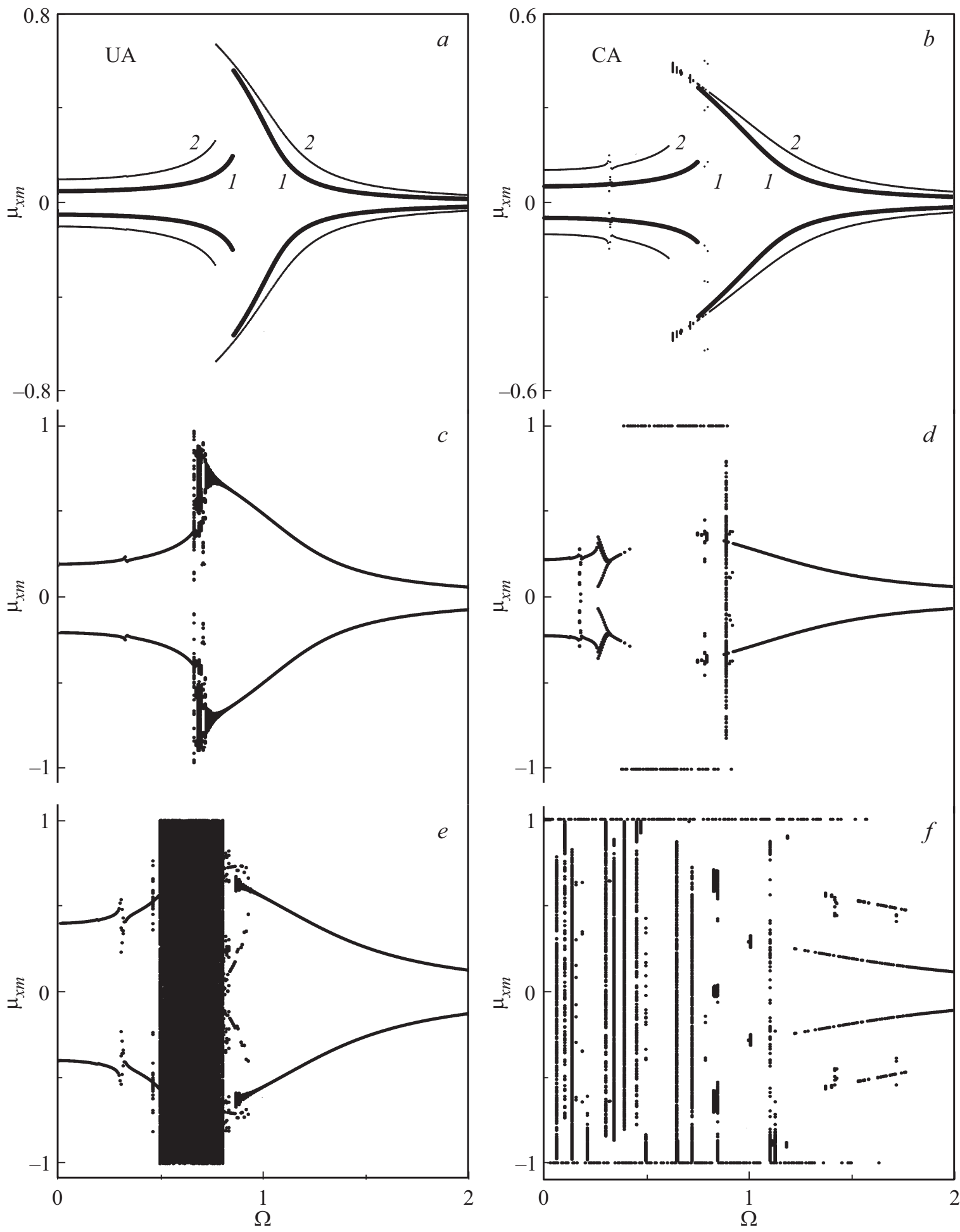

Рис. 1. Диаграммы зависимости экстремумов $x$-компоненты магнитного момента наночастицы с UA $(a, c, e)$ и CA - $(b, d, f)$ от частоты переменного поля $\Omega$ при его амплитуде $h_{0}=0.05,0.1$ ( $a$ и $b-$ кривые 1,2$), h_{0}=0.2(c, d), h_{0}=0.4(e, f)$; параметр диссипации $\alpha=0.01$.

где $k_{u}$ и $\mathbf{n}$ - константа UA и орт выделенного направления соответственно. Для СА каждая из трех компонент поля анизотропии определяется выражением

$$
H_{a v}=-\frac{2 V K_{1}}{m} \mu_{v}\left(1-\mu_{v}^{2}\right), \quad v \equiv x, y, z,
$$

где $\boldsymbol{\mu}=\mathbf{m} / m-$ нормированный магнитный момент, $V$ - объем наночастицы, а $K_{1}$ - константа СА. Введем безразмерные параметры: $\tau=\left(m \gamma / d^{3}\right) t, \quad$ где $d-$ диаметр наночастицы, внешнее поле $\mathbf{h}=\mathbf{H} d^{3} / m$, поле UA $\mathbf{h}_{a}=k_{u} \mathbf{n}(\boldsymbol{\mu} \mathbf{n}), \quad$ компоненты поля $\mathrm{CA}$ : 

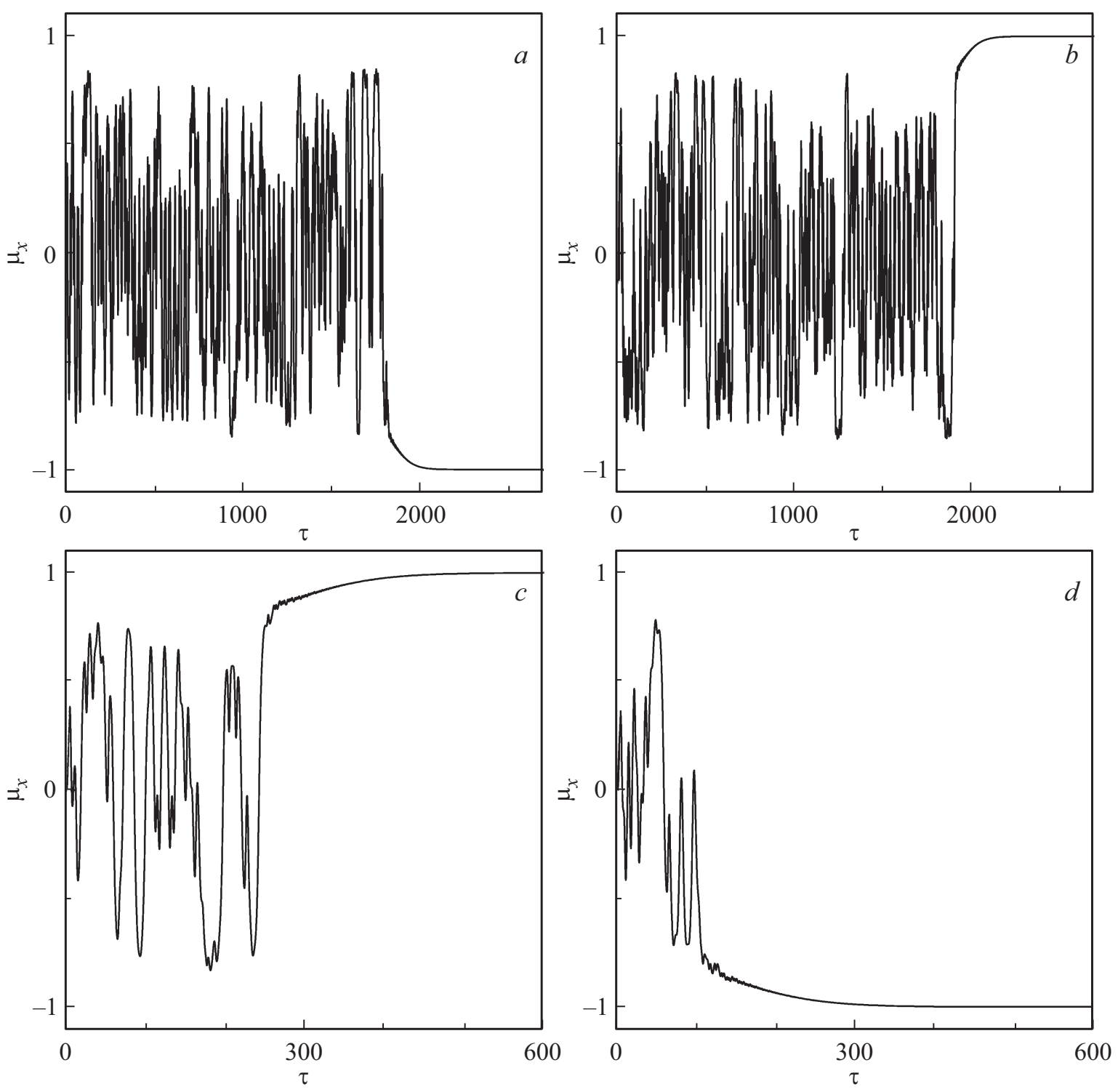

Рис. 2. Зависимость от времени $x$-компоненты магнитного момента наночастицы с СА при $90^{\circ}$-перемагничивании под воздействием поля с частотой $\Omega=0.5,0.6,0.7,0.8(a-d)$ и амплитудой $h_{0}=0.2$.

$h_{a v}=-2 k_{1} \mu_{v}\left(1-\mu_{v}^{2}\right)-\mathrm{c}$ константой $k_{1}=K_{1} V d^{3} / m^{2}$. Тогда уравнение (1) имеет вид

$$
\frac{\partial \boldsymbol{\mu}}{\partial \tau}=-\boldsymbol{\mu}\left(\mathbf{h}+\mathbf{h}_{a}\right)-\alpha \boldsymbol{\mu} \frac{\partial \boldsymbol{\mu}}{\partial \tau} .
$$

При дальнейшем анализе векторное уравнение (3) представляется тремя скалярными уравнениями. Так, уравнение для $x$-компонент имеет вид

$$
\begin{aligned}
& \left(1+\alpha^{2}\right) \frac{\partial \mu_{x}}{\partial \tau}=\left(\mu_{z}+\alpha \mu_{x} \mu_{y}\right)\left(h_{y}+h_{a y}\right) \\
& \quad-\left(\mu_{y}-\alpha \mu_{z} \mu_{x}\right)\left(h_{z}+h_{a z}\right)-\alpha\left(1-\mu_{x}^{2}\right)\left(h_{x}+h_{a x}\right) .
\end{aligned}
$$

Уравнения для остальных компонент имеют аналогичный вид. Равновесные ориентации и прецессионные динамические режимы магнитного момента определяются на основе численного анализа, который проводится с помощью метода Рунге-Кутта четвертого порядка.

\section{3. Динамика магнитного момента наночастицы}

Примем, что в исходной ориентации магнитный момент наночастицы ориентирован по оси $Y$ (то есть вдоль легкой оси UA или одной из легких осей $\mathrm{CA}$ ). При включении внешнего статического поля происходит перемагничивание наночастицы. Для случая СА с константой $k_{1}=-0.5$ и статического поля $h$, ориентированного вдоль оси $X$, при $h \approx 0.3$ начинается $90^{\circ}$-перемагничивание, затем - после быстрого выхода на насыщение (при $h \geq 0.5)$ - перемагничивание происходит по прямоугольной петле. Изменение на- 

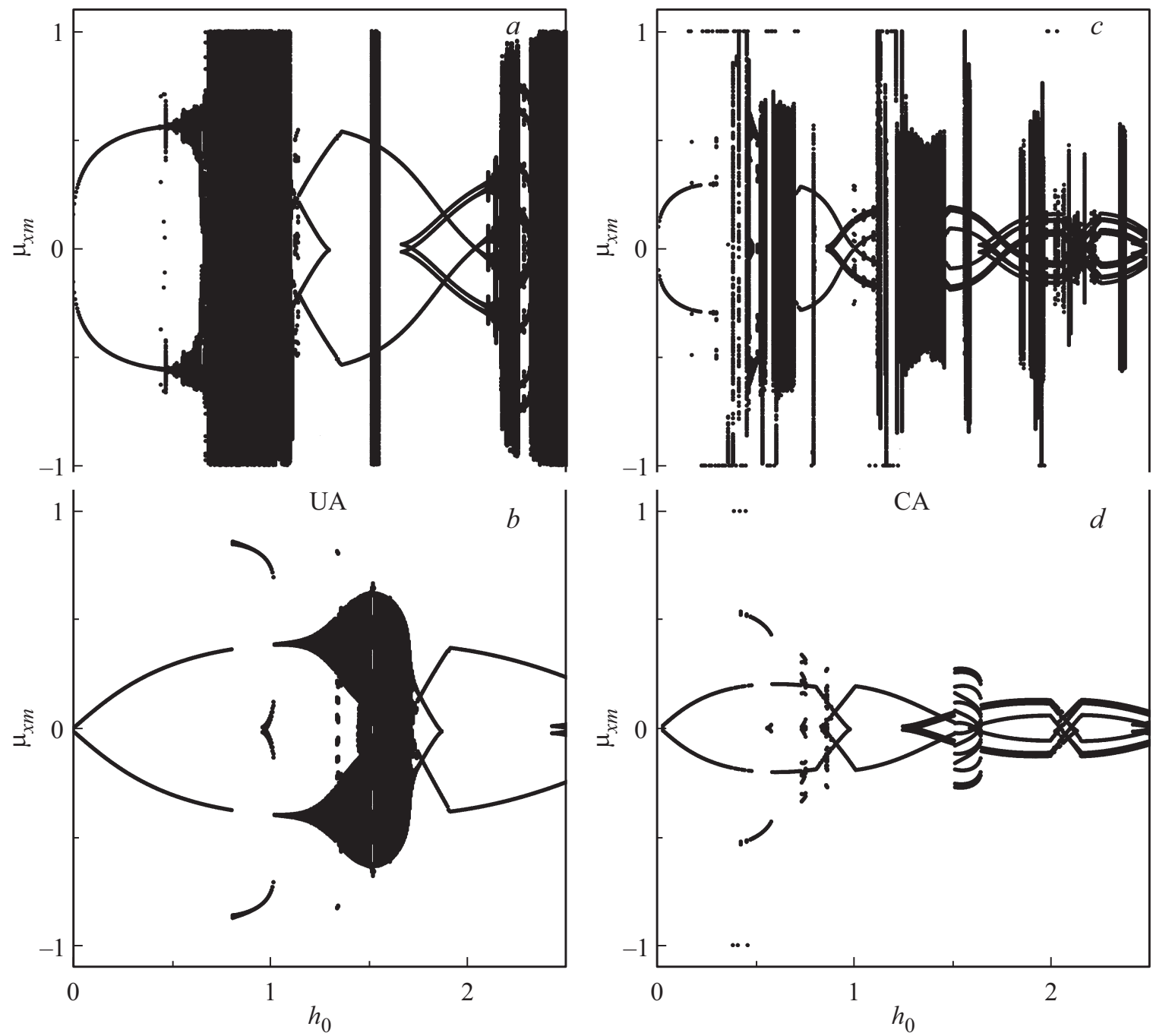

Рис. 3. Диаграмма зависимости экстремумов $x$-компонент магнитного момента наночастицы с UA $(a, b)$ и CA $(c, d)$ от амплитуды переменного поля при его частоте $\Omega=1(a, c)$ и $\Omega=1.5(b, d)$.

правления магнитного момента на противоположное, вслед за соответствующей переориентацией внешнего поля, происходит при $|h| \approx 1.2$. Петля гистерезиса для случая UA с константой $k_{u}=-1$ аналогична при ориентации внешнего поля вдоль оси $Y$ (за исключением $90^{\circ}$-перемагничивания). При дальнейшем анализе будем использовать указанные значения констант анизотропии $k_{u}$ и $k_{1}$.

Рассмотрим динамические прецессионные режимы магнитного момента, исходно ориентированного вдоль оси $Y$, под действием переменного магнитного поля $H=H_{0} \sin (\omega t)$, линейно поляризовано вдоль оси $X$. В приведенных величинах поле записывается в виде $\mathbf{h}=h_{0} \sin (\Omega \tau) \mathbf{e}_{x}$, где $\Omega=\left(d^{3} / m \gamma\right) \omega$. Анализ будем проводить с помощью бифуркационных диаграмм (БД), на которых каждому значению параметра отвечают максимальные и минимальные значения одной из компонент магнитного момента. Если значению параметра отвечают две или большее (но конечное) число точек, то при данном значении реализуются регулярные прецессионные режимы; множеству близко расположенных точек отвечает хаотическая или квазипериодическая динамика, единственной точке отвечает статическое (или близкое к нему) состояние $[18,19]$.

На рис. 1 приведена БД на плоскости $\left(\mu_{x m} ; \Omega\right)$, где каждому значению частоты переменного поля $\Omega$ отвечают максимальные и минимальные значения $x$-компоненты магнитного момента наночастицы. Диаграммы $(a, c, e)$ соответствуют наночастице с UA, a диаграммы $(b, d, f)$ - с СА. Амплитуда переменного поля принимается равной $h_{0}=0.05,0.1$ ( $a$ и $b-$ кривые 1,2$)$, $h_{0}=0.2(c, d), h_{0}=0.4(e, f)$. Для изолированного магнитного момента частота линейного резонанса $\omega_{0}=\gamma H$, что при переходе к безразмерным параметрам дает

$$
\Omega_{0}=\frac{d^{3} \omega_{0}}{m \mathcal{\gamma}}=h .
$$

При отсутствии подмагничивающего поля резонансная частота определяется только полем анизотропии и для 

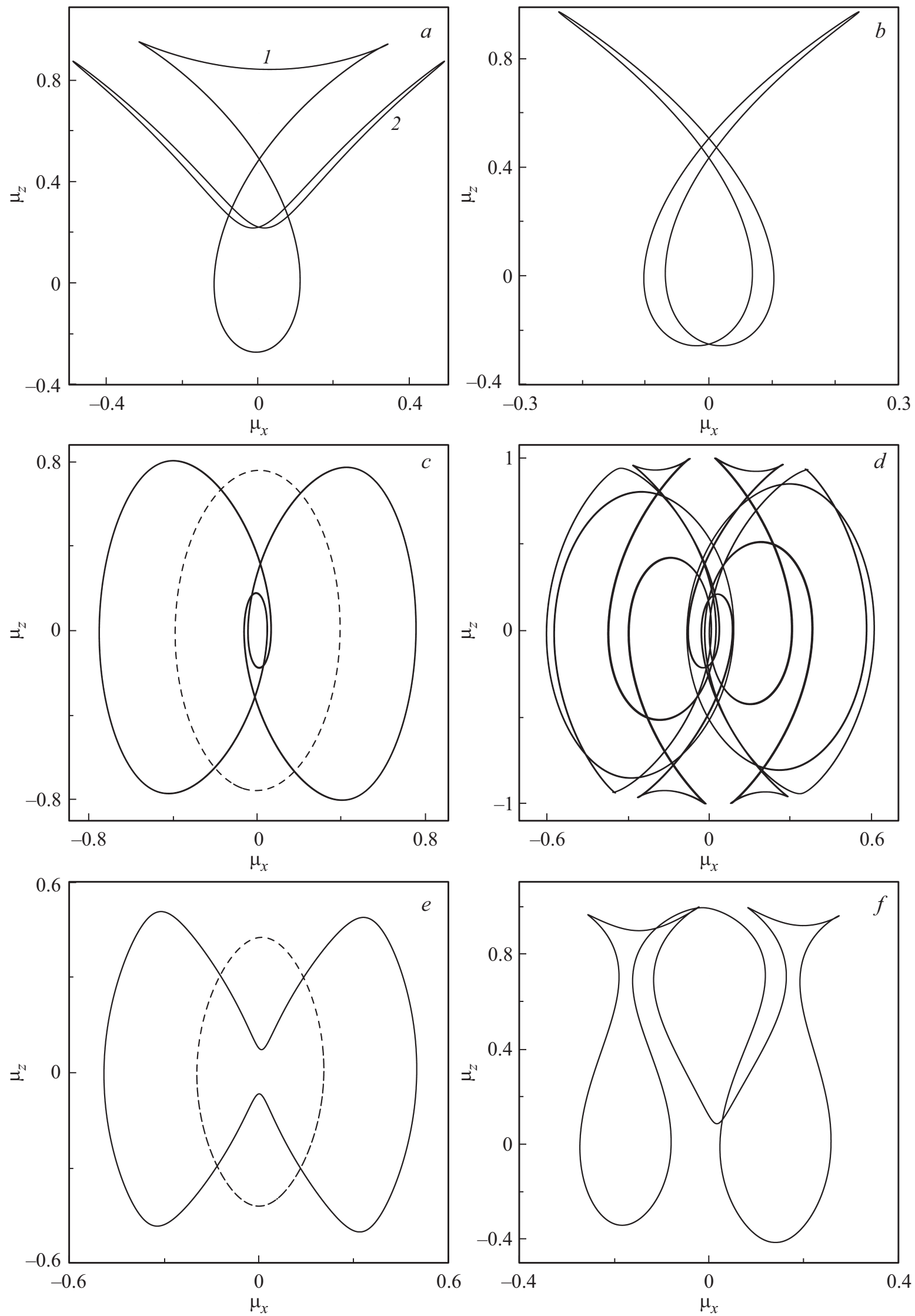

Рис. 4. Проекции траекторий регулярных колебательных режимов магнитного момента наночастиц с UA $(a-d)$ и CA - $(e, f)$ при действии поля с частотой $\Omega=1(a, b), \Omega=1.5(c-f)$ и амплитудой $h_{0}=1.2,1.5(a-$ кривые 1,2$), h_{0}=1.8,1.6,0.5,1.5(b-f)$; случаи $(c)$ и $(e)$ отвечают динамической биставильности. 

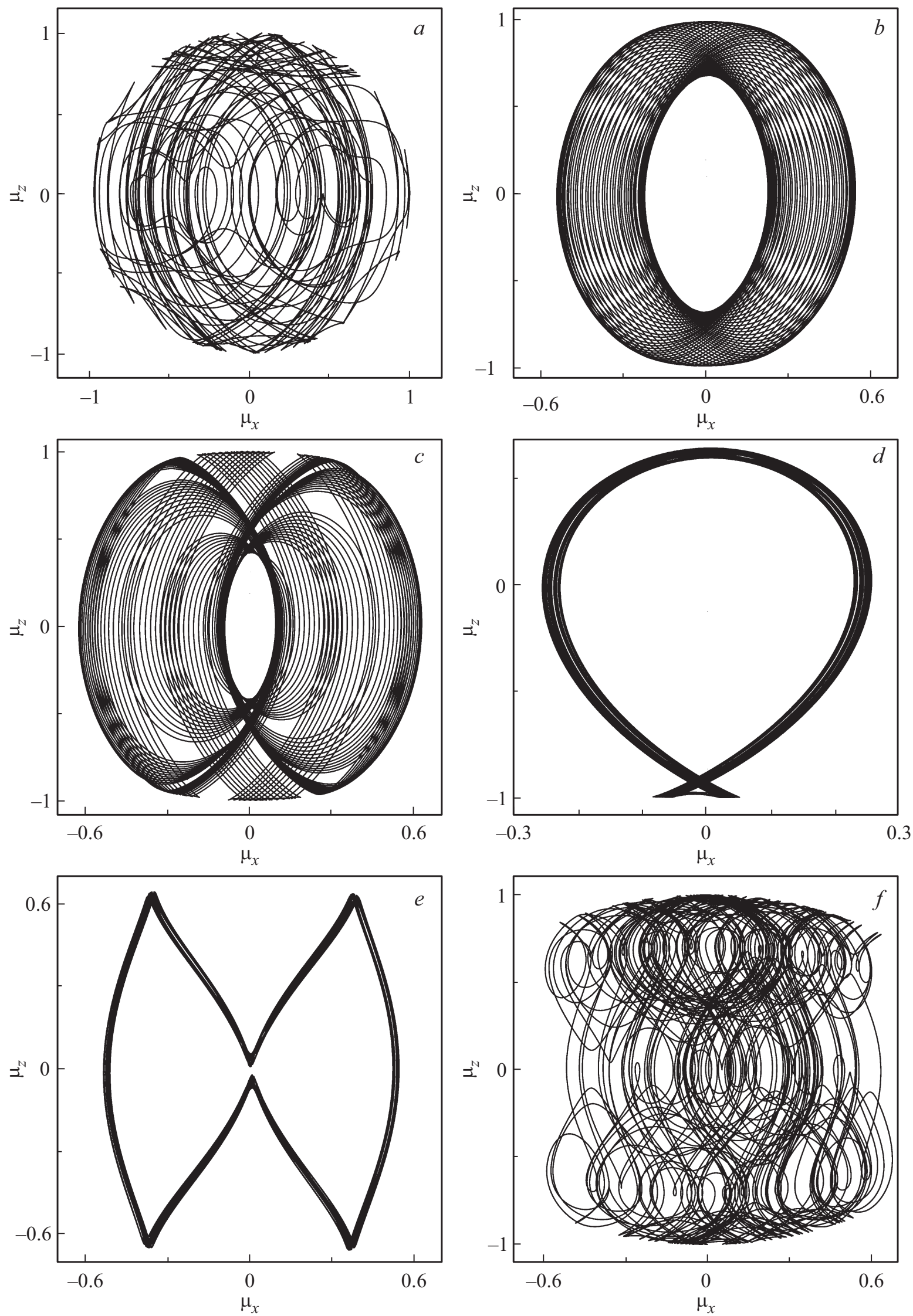

Рис. 5. Проекции аттракторов хаотических $(a, d-f)$ и квазипериодических $(b, c)$ режимов для магнитного момента наночастицы с $\mathrm{UA}(a-d)$ и с СА $(e, f)$ под действием поля с $\Omega=1(a, e, f), \Omega=1.5(b-d)$ и $h=0.7,1.5,1.7(a-d), h_{0}=0 . .5,0.7(e, f)$. 

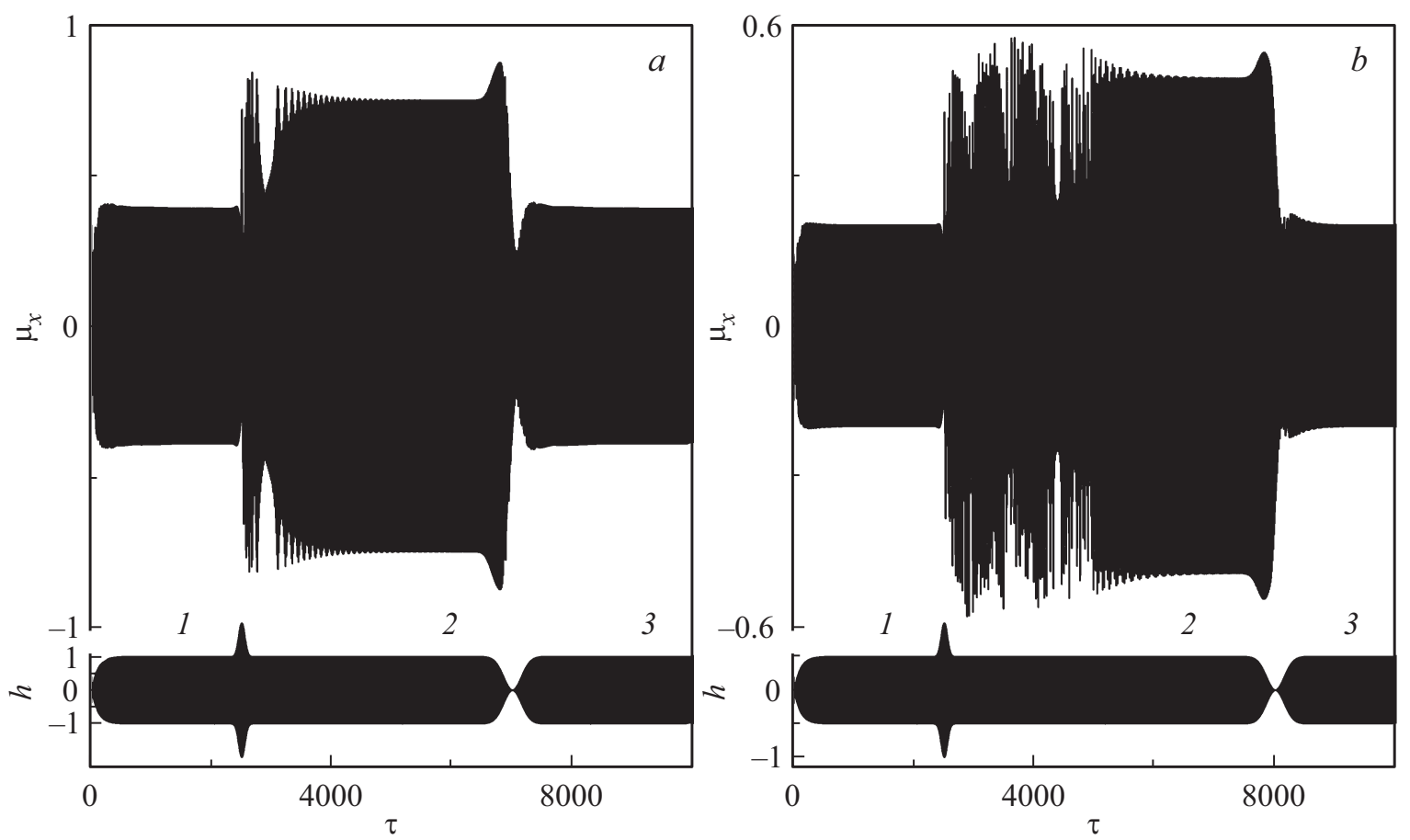

Рис. 6. Переход между колебательными режимами при наличии динамической бистабильности для наночастицы с UA $(a)$ и CA $(b)$ за счет импульсного изменения амплитуды переменного поля (показанного в нижней части рисунка); параметры переменного поля $\Omega=1.5, h_{0}=0.5,1(b, a)$.

наночастиц с UA равна $\Omega=k_{u}$, а для наночастиц с СА $\Omega=2\left|k_{1}\right|[17]$. Однако, как видно из приведенных БД уже при достаточно малых амплитудах переменного поля $(a, b)$ резонанс является нелинейным, при этом с увеличением амплитуды поля резонансная частота смещается в сторону меньших значений. При дальнейшем увеличении переменного поля возникают частотные области, отвечающие более сложным регулярным, а также квазипериодическим колебаниям или хаотической динамике $(c, e)$, что наиболее характерно для наночастиц c UA. В случае же наночастиц с CA характерным является появление частотных областей, отвечающих за $90^{\circ}$-перемагничивание: под воздействием переменного поля после переходного процесса магнитный момент ориентируется вдоль оси $X$.

На рис. 2 приведена зависимость от времени $x$-компоненты магнитного момента наночастицы с СА при $90^{\circ}$-перемагничивании полем с амплитудой $h_{0}=0.2$ и частотой $\Omega=0.5,0.6,0.7,0.8(a-d)$. В большинстве случаев увеличение частоты поля приводит к уменьшению длительности переходного процесса, однако это не всегда справедливо $(a, b)$, так как данное перемагничивание имеет вероятностный характер и длительность переходных колебаний может быть различной.

На рис. 3 приведены БД, на которых параметром является амплитуда переменного поля. Одноосной и кубической анизотропиям отвечают соответственно диаграммы $(a, b)$ и $(c, d)$ при частоте поля $\Omega=1(a, c)$ и $\Omega=1.5(b, d)$. На диаграммах $(a-c)$ имеют место как интервалы поля, отвечающие различным по форме регулярным колебательным режимам, так и интервалы, отвечающие хаотической или квазирегулярной динамике. На диаграммах $(b, d)$ имеют место интервалы значений $h_{0}$, отвечающие динамической бистабильности, когда при одних и тех же параметрах системы могут реализоваться два (или более) различных динамических режима. Реализация того или иного режима зависит от начального состояния динамической системы. На приведенных диаграммах бистабильное состояние проявляется прерыванием одной ветви колебательных режимов, и началом другой ветви, а далее снова появлением первой ветви. Это объясняется тем, что при каждом значении параметра исходной являлась одна и та же ориентация магнитного момента, а вероятность реализации того или иного колебательного режима (обусловленная, в частности, величиной притягивающей к аттрактору области фазового пространства) меняется с изменением величины параметра.

Проекции аттракторов регулярных режимов на плоскость $(x z)$ приведены на рис. 4. Наночастицам с UA отвечают режимы $(a-d)$, а с $\mathrm{CA}-(e, f)$. Колебания магнитного момента устанавливаются под воздействием переменного поля с частотой $U=1(a, b), \Omega=1.5(c-f)$ и амплитудой $h_{0}=1.2,1.5(a-$ кривые 1,2$), h_{0}=1.8$, $1,1.6,0.5,1.5(b-f)$. Случаи $(c)$ и $(e)$ отвечают динамической бистабильности: здесь режимы, представленные пунктирными кривыми, реализуются при тех же соответствующих параметрах, но при других начальных услови- 

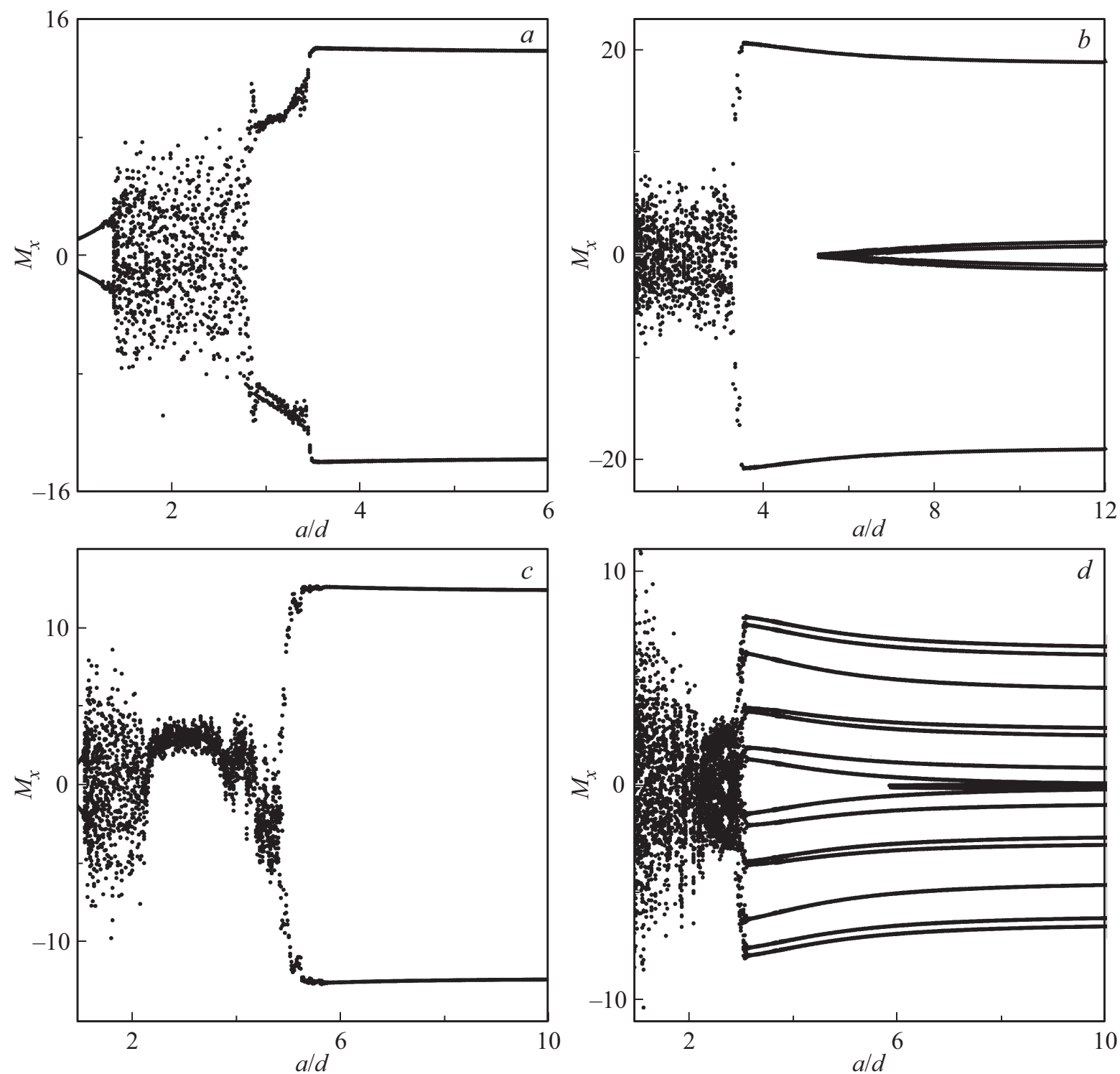

Рис. 7. Диаграммы зависимости динамических режимов суммарного магнитного момента решетки $5 \times 5$ наночастиц с UA $(a, b)$ и СА $(c, d)$ от нормированного расстояния между наночастицами; параметры поля, поляризованного перпендикулярно плоскости решетки: $\Omega=1(a), \Omega=1.5(b-d), h_{0}=0.4,0.5,1,1.6(c, a, b, d)$.

ях (более медленном нарастании амплитуды переменного поля). Видно, что при разных значениях параметров переменного поля устанавливаются различные (как по амплитуде, так и по форме аттракторов) регулярные колебательные режимы магнитного момента наночастицы. Период данных колебаний во многих случаях кратен периоду переменного поля.

На рис. 5 приведены проекции аттракторов хаотических $(a, d-f)$ и квазипериодических $(b, c)$ динамических режимов для магнитного момента наночастицы с UA $(a-d)$ и с CA $(e, f)$, реализуемых под действием переменного поля с частотой $\Omega=1(a, e, f)$, $\Omega=1.5(b-d)$ и амплитудой $h_{0}=0.7,1.4,1.5,1.7(a-d)$, $h_{0}=0.5,0.7(e, f)$. Видно, что хаотичность динамики может иметь различную степень проявления. В случа- ях $(a, f)$ сложные хаотические колебания имеют двумерный аттрактор с большой площадью, в случаях же $(d, e)$ хаотичность отражается только в размывании аттрактора, близкого к регулярному. При квазипериодических колебаниях аттракторы имеют форму тора.

Переход между двумя колебательными режимами при наличии динамической бистабильности представлен на рис. 6, где показана зависимость от времени $x$-компоненты магнитного момента наночастицы c UA $(a)$ и CA $(b)$. Переменное поле имеет параметры $\Omega=1.5$ и $h_{0}=0.5,1(b, a)$. Для перехода с одного колебательного режима на другой используется импульсное увеличение амплитуды переменного поля, а для возвращения к первому режиму - импульсное ее уменьшение. При этом временная огибающая переменного поля име- 
ет вид гауссовых импульсов (на пьедестале)

$$
h_{s}(\tau)=h_{0}+h_{i} \exp \left(-\left[\tau-\tau_{i}\right]^{2} / 2 \tau_{0}^{2}\right),
$$

где $h_{i}, \tau_{i}$ и $\tau_{0}-$ амплитуда, временной сдвиг максимума и длительность наложенного импульса. Для увеличения амплитуды поля и перехода с колебательного режима 1 на режим 2, имеющий бо́льшую амплитуду колебаний, использованы следующие параметры импульса: $h_{i}=0.5,1(b, a), \tau_{i}=2500$, и $\tau_{0}=50$. Для возвращения к первоначальному режиму (с режима 2 на режим 3 ) использовались параметры импульса: $h_{i}=0.5,1(b, a)$, $\tau_{i}=7000,8000(a, b)$, и $\tau_{0}=150$. Таким образом для обоих типов наночастиц может быть реализован динамический переход между колебательными режимами, входящими в бистабильное состояние.

\section{4. Динамика магнитного момента решетки наночастиц}

Исследуем регулярную плоскую решетку $5 \times 5$ однодоменных наночастиц при различных значениях параметра решетки а для выявления условий, когда взаимным влиянием магнитных моментов частиц друг на друга можно пренебречь. В этом случае динамические режимы суммарного магнитного момента $\mathbf{M}=\Sigma \boldsymbol{\mu}_{i}$ решетки должны быть аналогичны режимам изолированных наночастиц. В общем случае все диполи связаны диполь-дипольным взаимодействием. Эффективное поле, действующее на $i$ диполь со стороны остальных диполей, определяется выражением

$$
\mathbf{H}_{i}^{\mathrm{ef}}=\sum_{n \neq i}\left[\frac{3\left(\mathbf{m}_{n} \mathbf{r}_{i n}\right) \mathbf{r}_{i n}-\mathbf{m}_{n} r_{i n}^{2}}{r_{i n}^{5}}\right],
$$

где $\mathbf{r}_{i n}$ и $r_{i n}-$ радиус-вектор и расстояние между $i$ - и $n$-диполями. При переходе к безразмерным параметрам: $\mathbf{e}_{i n}=\mathbf{r}_{i n} / r_{\text {in }}$ получаем выражение:

$$
\mathbf{h}_{i}^{\mathrm{ef}}=\sum_{n \neq i}\left[\frac{3\left(\boldsymbol{\mu}_{n} \mathbf{e}_{i n}\right) \mathbf{e}_{i n}-\boldsymbol{\mu}_{n}}{l_{i n}^{3}}\right],
$$

которое должно быть добавлено в уравнения (3) и (4). Динамические режимы суммарного магнитного момента системы будем искать при учете связи всех наночастиц решетки друг с другом.

На рис. 7 представлены БД динамических режимов суммарного магнит- ного момента решетки $5 \times 5$ наночастиц с UA $(a, b)$ и CA $(c, d)$, где параметром является нормированный параметр решетки $a / d$. Частота переменного поля, линейно поляризованного перпендикулярно плоскости решетки (вдоль оси $X$ ), $\Omega=1(a), \Omega=1.5(b-d)$, его амплитуда $h_{0}=0.4,0.5$, $1,1.6(c, a, b, d)$. Видно, что при близком расположении элементов решетки $(a / d \approx 1)$ в некоторых случаях устанавливаются регулярные низкоамплитудные колебания $(a, c)$, в остальных случаях имеет место хаотическая динамика. При удалении элементов решетки друг от друга устанавливаются различные хаотические колебания. Когда же расстояние между элементами $a / d \geq 6-10$ диполь-дипольное взаимодействие слабо влияет на динамику магнитных моментов и колебания каждого из них близко к колебаниям магнитного момента изолированной наночастицы.

Приведем переход от безразмерных величин к размерным для наночастиц железа: магнитный момент $m \approx 3 \mu_{B} N$, где $N$ - число атомов в наночастице, ее диаметр $D \approx A(6 N / \pi)^{1 / 3}, A=0.286$ - параметр кристаллической решетки. Для $N=512$ ее размер составляет $D \approx 2.8 \mathrm{~nm}$, а $m \approx 1.4 \cdot 10^{-17} \mathrm{erg} /$ Ое. С учетом $\gamma=1.76 \cdot 10^{7}(\mathrm{Oe} \cdot \mathrm{s})^{-1}$ получаем следующие численные оценки для времени $t=\left(d^{3} / \gamma m\right) \tau \approx 8.8 \cdot 10^{-11} \tau \mathrm{s}$, частоты $\omega=\left(\gamma m / d^{3}\right) \Omega \approx 1.1 \cdot 10^{10} \Omega \cdot \mathrm{s}^{-1}$, магнитного поля $H=\left(m / d^{3}\right) h \approx 647 h \mathrm{Oe}$ и константы СА $K_{1}=\left(\mathrm{m}^{2} / V d^{3}\right) k_{1} \approx 8.0 k_{1} \cdot 10^{6} \mathrm{erg} / \mathrm{cm}^{3}$.

\section{5. Заключение}

Исследование динамики магнитного момента наночастицы с одноосной и кубической анизотропиями, показало, что в отсутствии подмагничивающего поля резонансная частотная область определяется полем анизотропии. При рассмотрении широкого диапазона частот и амплитуд переменного поля и при построении бифуркационных диаграмм выявлены условия реализации различных регулярных колебательных режимов, отличающихся как по форме аттракторов, так и по амплитуде и периоду колебаний. Обнаружены также хаотические динамические режимы с различной степенью проявления хаотизации, а так же режимы квазипериодических колебаний. В случае кубической анизотропии найдены интервалы частоты переменного поля, при которой реализуется динамическое $90^{\circ}$-перемагничивание наночастицы на направление поляризации переменного поля. Из бифуркационных диаграмм выявлены также состояния динамической бистабильности, когда при одних и тех же параметрах системы и поля могут устанавливаться два различных колебательных режима, на реализации которых влияют начальные условия, в частности, скорость нарастания амплитуды переменного поля. Получены переходы между входящими в бистабильность колебательными режимами с помощью импульсного увеличения и уменьшения амплитуды переменного поля.

При рассмотрении решеток наночастиц, связанных диполь-дипольным взаимодействием показано, что в случае достаточно малого расстояние между частицами достаточно мало $(a / d \leq 4-6)$ устанавливаются или низкоамплитудные колебания суммарного магнитного момента решетки, или хаотическая динамика. При $a / d \geq 6-10$ диполь-дипольное взаимодействие слабо влияет на колебательные режимы магнитных моментов отдельных наночастиц и для решетки в целом устанавливаются динамические режимы, характерные при данных параметрах внешнего поля для изолированных наночастиц. 


\section{Список литературы}

[1] R. Skomski. J. Phys.: Condens. Matter. 15, R841 (2003).

[2] Е.3. Мейлихов, Р.М. Фарзетдинова. ЖЭТФ 122, 1027 (2002).

[3] П.В. Бондаренко, А.Ю. Галкин, Б.А. Иванов. ЖЭТФ 139, 1127 (2011).

[4] А.А. Фраерман. УФН 182, 1345 (2012).

[5] С.А. Дзян, Б.А. Иванов. ЖЭТФ 142, 969 (2012).

[6] Е.3. Мейлихов, Р.М. Фарзетдинова. ФТТ 56, 2326 (2014).

[7] Ю.П. Иванов, А.И. Ильин, Е.В. Пустовалов, Л.А. Чеботкеич. ФТТ 52, 1576 (2010).

[8] В.А. Кособукин, Б.Б. Кричевцов. ФТТ 52, 759 (2010).

[9] С.А. Гусев, Ю.Н. Ноздрин, М.В. Сапожников, А.А. Фраерман. УФН 170, 331 (2000).

[10] Л.Н. Котов, Л.С. Носов, Ф.Ф. Асадуллин. ЖТФ 78, 60 (2008).

[11] E. De Biasi, E. Lima (Jr), C.A. Ramos A. Butera, R.D. Zysler. J. Magn. Magn. Mater. 326, 138 (2013).

[12] И.Р. Каретникова, И.М. Нефедов, М.В. Сапожников, А.А. Фраерман, И.А. Шеремевский. ФТТ 43, 2030 (2001).

[13] A.M. Shutyi, S.V. Eliseeva, D.I. Sementsov. Phys. Rev. B 91, 024421 (2015)

[14] A.M. Shutyi, D.I. Sementsov. J. Magn. Magn. Mater. 401, 1033 (2016).

[15] А.М. Шутый. ЖЭТФ 145, 1048 (2014).

[16] А.М. Шутый, Д.И. Семенцов. Письма в ЖЭТФ 99, 806 (2014).

[17] А.Г. Гуревич, Г.А. Мелков. Магнитные колебания и волны, Наука, М. (1994).

[18] А.Ю. Лоскутов, А.С. Михайлов. Основы теории сложных систем. НИЦ „РХД“, Ижевск (2008).

[19] Д.И. Семенцов, А.М. Шутый. УФН 177, 831 (2007).

Редактор Ю.Э. Китаев 